\title{
Edge detection based on augmented lagrangian method for low- quality medical images
}

\author{
Vo Thi Hong Tuyet ${ }^{1 *}$ \\ ${ }^{1}$ Ho Chi Minh City Open University, Vietnam \\ *Corresponding author: tuyet.vth@ou.edu.vn
}

\begin{abstract}
ARTICLE INFO
ABSTRACT

DOI: $10.46223 /$ HCMCOUJS. tech.en.8.1.910.2018

Medical images are useful for the treatment process. They contain a lot of information on displaying abnormalities in your body. The contour of medical images is a matter of interest. In there, edge detection is a process prepared for boundaries.

Received: August $4^{\text {th }}, 2017$ Therefore, the edge detection of medical images is very important.

Revised: November $17^{\text {th }}, 2017$ Other previous methods must sacrifice time for the accurate Accepted: April 16 ${ }^{\text {th }}, 2018$ results. It is because the medical images in the real world have many impurities. In this paper, I propose a method of detecting edges in medical images which have impurities by using augmented lagrangian method to improve the Canny algorithm.

Keywords:

augmented lagrangian method, canny, edge detection My algorithm improves the ability to detect edges faster. Compared with other recent methods, the proposed method is more efficient.
\end{abstract}

\section{Introduction}

When medical images have impurities, blur and noise details, the quality of them become worse. This is a big problem for health professionals to diagnose a disease. Because the treatment processing is based on the information from medical images. They provide many details of an inside human body that naked eyes cannot see. The size or location of each part of a body changes with the initial manifestation of diseases. The clearer the contours of an object (e.g., bone, liver, blood vessels, etc...) is, the higher the quality of medical images is. So detecting abnormalities accurately is very useful to maintain patients' lives. The detection of a body part is a process that combines pixels together, and it is called edge detection. If an algorithm detects many edges, we will have more information about objects and we will be more comfortable in contour detecting or segmentation.

The principles of edge detection methods usually include removing the noise as the first step in this process (Marr \& Hildreth, 1980). The quality of medical images is almost worse because of many reasons. The noised pixels which make the first step of edge detection don't get good results because of the presence of a weak object in input images. Therefore, medical images that have weak objects become a big challenge for edge detection.

In the past, there are many algorithms which are proposed for edge detection, such as Canny (Canny, 1986), Sobel (Vincent \& Folorunso, 2009), B-spline (Bhatt \& Warkhedkar, 
2008; Yuping \& Yuanlong, 1995) or in the generation types of wavelet transform (Easley, Labate, \& Lim, 2008; Yuping \& Yuanlong, 1995; Zhang \& Bao, 2002). However, the previous methods are limited to weak objects. Wavelet transform could overcome the disadvantage, but the time processing is very long.

In this paper, I propose an algorithm for edge detection of objects in low-quality medical images. I present the basis of the augmented lagrangian method and edge detection in section 2. The proposed method is presented in section 3. The results of the experiments are compared with the ones of the recent methods in section 4 . Finally, the conclusions are shown in section 5 .

\section{Augmented lagrangian method and edge detection method}

\subsection{Augmented lagrangian method}

Image restoration is an algorithm that must recover the sharp image. In this process, the rehabilitation vector is a difficult problem. The idea of minimizing a total variation on the optimization problem for spatial-temporal data (Stanley, Khoshabeh, Kristofor, Philip, \& Truong, 2011) was proposed. They used an Augmented Lagrangian Method (ALM) to solve the constrained problem.

A vector denoting the unknown (potentially sharp) image which has a size $\mathrm{Mx} N$ is called $\mathrm{f}$ ( $\mathrm{f} \in R^{M N \times 1}$ ). $\mathrm{f}$ is an ingredient of the equation to find the observed image $\mathrm{g}, \mathrm{g} \in R^{M N \times 1}$. It is a linear shift-invariant imaging system to calculate as:

$$
\mathrm{g}=\mathrm{Hf}+\eta
$$

where $\mathrm{f}$ is a vector denoting the unknown (potentially sharp) image of size $\mathrm{Mx} \mathrm{N}, \mathrm{g}$ is a vector denoting the observed image, $\eta \in R^{M N \times 1}$ is a vector denoting the noise/blur, and the matrix $\mathrm{H} \in R^{M N \times M N}$ is a linear transformation representing convolution operation.

TV/L1 minimization (for denoising image) and TV/L2 minimization (for deblurring image) were two problems are mentioned in ALM (Stanley et al., 2011). They were defined as:

$$
\begin{aligned}
& \underset{\mathrm{f}}{\operatorname{minimize}} \frac{\mu}{2}\|H f-g\|^{2}+\|f\|_{T V} \\
& \underset{\mathrm{f}}{\operatorname{minimize}} \mu\|H f-g\|_{1}+\|f\|_{T V}
\end{aligned}
$$

With the equations, $\mu$ is the regularization parameter. The authors were to find a saddle point of L(f, $u, y)$. Then, they used the alternating direction method (ADM) to solve fsubproblem, u-subproblem with TV/L2 and f-subproblem, u-subproblem and r-subproblem with TV/L1. The equation as:

$$
\underset{\mathrm{f}, \mathrm{u}}{\operatorname{minimize}} \frac{\mu}{2}\|\mathrm{Hf}-\mathrm{g}\|^{2}+\|\mathrm{u}\|_{1}
$$

and

$$
\underset{f, r, u}{\operatorname{minimize}} \mu\|r\|_{1}+\|\mathrm{u}\|_{1}
$$

Subject to $r=\mathrm{Hf}-\mathrm{g}$ and $\mathrm{u}=\mathrm{Df}$. 
ALM (Stanley et al., 2011) can be summarized as follows:

(i) Input: vector denoting the observed image and convolution matrix, regularization parameter, the isotropic total variation;

(ii) Set parameter with value default. This step is depended on other types of TV/L1 or TV/L2;

(iii) Initialize for the first value, such as $f, u$;

(iv) Compute the matrices of the first-order forward finite difference operators along with the horizontal, vertical and temporal directions;

(v) With not coverage do:

+ Solve the subproblems and update parameters;

+ Check convergence, if false continue.

This proposed method is also used for video restoration. Because the truth of each video is still the sum of many images.

\subsection{Edge detection method}

Edge detection is a process that finds the connection between pixels. The connection depends on many reasons, such as distance, the colors between two different regions in an image, shadows, depth, texture, or surface color, etc. Sometimes, this process is also based on the important features of edges.

Edge is the boundary of regions in an image. The boundary may create many lines, corners, curves in an image because of intensity changes.

The definition of edge detection was proposed in 1980. The sequence includes smoothing, enhancement, detection and localization (Marr \& Hildreth, 1980). The first step is the denoising for input images. The important requirement is to keep the features of edges when removing noise. Then, the authors use filters to improve the quality of the edges. The detection is the continued step, it is based on the values of threshold (the results of the previous step which are enhanced by filters). The final step is the localization - based on the location of each edge.

In the continuation of the idea about edge detection, John Canny proposed Canny method in 1986 with the name: "A computational approach to edge detection". In 1987, R. Deriche used Canny to derive a recursively implemented optimal edge detector. The canny method used a Gaussian filter to smooth the image and applied the double threshold to determine potential edges. That parallel, Sobel (Vincent \& Folorunso, 2009) was based on the approximation values $\mathrm{M}_{\mathrm{x}}$ and $\mathrm{M}_{\mathrm{y}}$ :

$$
M_{x}=\left[\begin{array}{ccc}
-1 & 0 & 1 \\
-2 & 0 & 2 \\
-1 & 0 & 1
\end{array}\right] \text { and } M_{y}=\left[\begin{array}{ccc}
-1 & -2 & -1 \\
0 & 0 & 0 \\
1 & 2 & 1
\end{array}\right]
$$

B-spline was also detected for edges (Bhatt \& Warkhedkar, 2008; Yuping \& Yuanlong, 1995) or combined with Active contour Snakes (Brigger \& Unser, 1998). Other algorithms 
were detected in wavelet transform (Yuping \& Yuanlong, 1995; Zhang \& Bao, 2002). With transform, the authors proposed the detection based on the combination between pixels in domain. Edge detection is based on estimating the gradient: strength, gradient direction by -90 degrees. The gradient is the two-dimensional equivalent of the first derivative and is defined as the vector.

\section{Edge detection based on Augmented Lagrangian method to improve the results of Canny method}

Every little detail of medical images is very important. Each detail is an area that is connected by edges. Therefore, the number of edge detection is very helpful for the treatment of specialists in this field. When edge detection exacts the contour of each part, the change of size, shape, etc... maybe a manifestation of a disease.

Many methods are based on masks such as Sobel (Vincent \& Folorunso, 2009) and the proposed method takes advantage of the structure in local images. Recently, the transform is continued to solve this problem (Yuping \& Yuanlong, 1995; Zhang \& Bao, 2002). But, with transform, images must be adapted for threshold and filter many times. That loses small details which are extremely precious in medical images. And applications take a lot of time.

That doesn't yet mention that the main reason why medical images with noise make a weak object. The detection of noisy images by Canny has a stronger contour than Sobel because it tracks edge by hysteresis.

Canny algorithm (Canny, 1986) includes 5 steps: smooth images - find the intensity edge detection by non-maximum suppression - to determine potential edges - track edges. It is similar to using threshold and filter in transform. The idea may lose the information of images because of applying the double threshold to determine potential edges. The process is very complex by Gaussian filter and threshold.

In this paper, I accost the Augmented Lagrangian Method (ALM) to improve the result of the Canny method. My algorithm can be summarized in Figure 1. 


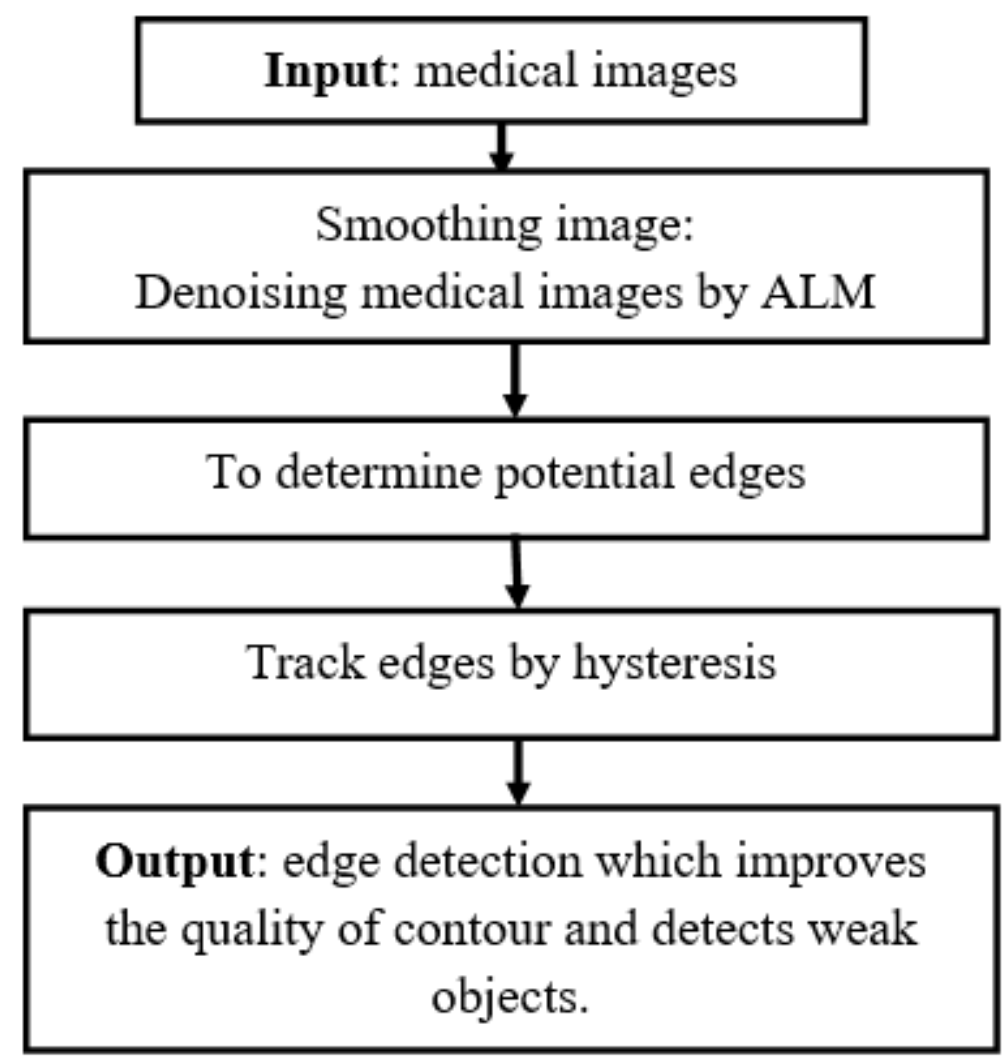

- The smoothing image:

Figure 1. The proposed method

ALM uses minimization to remove the noise or blur details out of pixels. Here, my proposed method applies the TV/L1 process which follows (Stanley et al., 2011):

$$
\text { minimize }_{f, r, u} \mu\|r\|_{1}+\|u\|_{1}
$$

(i) Input: vector denoting the observed image $(\mathrm{g})$ and convolution matrix $(\mathrm{H})$, regularization parameter $\mu$, the isotropic total variation $\beta_{x}, \beta_{y}, \beta_{t}$;

(ii) Set parameter with value default for $\rho_{r}=2, \rho_{0}=100\left(\rho_{r}\right.$ is a regularization parameter) and $\alpha_{0}=0.7$;

(iii)Initialize $\mathrm{f}_{0}=\mathrm{g}, \mathrm{u}_{0}=\mathrm{Df}_{0}, \mathrm{y}_{0}=0, \mathrm{r}_{0}=\mathrm{Hf}_{0}-\mathrm{g}, \mathrm{z}_{0}=0$. ( $\mathrm{y}$ is the Lagrange multiplier);

(iv)Compute the matrices of the first-order forward finite difference operators along with the horizontal, vertical and temporal directions.

while (not coverage) do:

- Solve the f-subproblem is:

$$
\text { minimize }_{f}=\frac{\rho_{0}}{2}\|r-H f+g\|^{2}+\frac{\rho_{r}}{2}\|u-D f\|^{2}+z^{T} H f+y^{T} D f
$$

where $D=\left[\begin{array}{ccc}D_{x}^{T} & D_{y}^{T} & D_{t}^{T}\end{array}\right]^{T}, \mathrm{D}_{\mathrm{x}}, \mathrm{D}_{\mathrm{y}}, \mathrm{D}_{\mathrm{t}}$ are the first-order forward finite-difference operators along with the horizontal, vertical, and temporal directions. 
f-subproblem is improved by the equation:

$$
f=\mathcal{F}^{-1}\left[\frac{\mathcal{F}\left[\rho_{0} H^{T} g+H^{T}\left(\rho_{0} r-z\right)+D^{T}\left(\rho_{r} u-y\right)\right]}{\rho_{0}|\mathcal{F}[H]|^{2}+\rho_{r}\left(\left|\mathcal{F}\left[D_{x}\right]\right|^{2}+\left|\mathcal{F}\left[D_{y}\right]\right|^{2}+\left|\mathcal{F}\left[D_{t}\right]\right|^{2}\right)}\right]
$$

where $\mathcal{F}$ denotes the three-dimensional Fourier Transform operator.

- Solve the u-subproblem is:

$$
u_{k+1}=\underset{u}{\arg \min }\|u\|_{1}-y_{k}^{T}\left(u-D f_{k+1}\right)+\frac{\rho_{r}}{2}\left\|u-D f_{k+1}\right\|^{2}
$$

by equation:

$$
u_{x}=\max \left\{\left|v_{x}\right|-\frac{1}{\rho_{r}}, 0\right\} * \operatorname{sign}\left(v_{x}\right)
$$

- Solve the r-subproblem:

$$
\text { minimize }_{r} \quad \mu\|r\|_{1}-z^{T} r+\frac{\rho_{0}}{2}\|r-H f+g\|^{2}
$$

by equation:

$$
r=\max \left\{\left|H f-g+\frac{1}{\rho_{0}} z\right|-\frac{\mu}{\rho_{0}}, 0\right\} * \operatorname{sign}\left(H f-g+\frac{1}{\rho_{0}} z\right)
$$

- Update the Lagrange multiplier y and z:

$$
y_{k+1}=y_{k}-\rho_{r}\left(u_{k+1}-D f_{k+1}\right)
$$

and

$$
z_{k+1}=z_{k}-\rho_{0}\left(r_{k+1}-H f_{k+1}+g\right)
$$

- Update:

$$
\rho_{r}=\left\{\begin{array}{l}
\gamma \rho_{r}, i f\left\|u_{k+1}-D f_{k+1}\right\|_{2} \geq \alpha\left\|u_{k}-D f_{k}\right\|_{2} \\
\rho_{r}, \text { otherwise }
\end{array}\right.
$$

- Check convergence: if $\left\|f_{k+1}-f_{k}\right\|_{2} /\left\|f_{k}\right\|_{2} \leq$ tol then break end while.

\section{- To determine potential edges and track edges:}

After the smoothing step by ALM, the determining gradients of the medical images is also known as the edge strengths. This value is calculated by Euclidean distance measure, and it will be used to define edges to be shown. Euclidean distance can calculate by:

$$
E=\left(\sum_{i=1}^{n}\left|x_{i}-y_{i}\right|^{2}\right)^{1 / 2}
$$

where $\mathrm{x}$ and $\mathrm{y}$ are the coordinates of image pixels.

Nonetheless, these edges will be converted to "sharp" edges in the non-maximum suppression step. In each pixel of the gradient image, the gradient direction $<45^{\circ}$ and 8 connected neighborhoods are mentioned. The direction of edges is shown by the equation: 


$$
\theta=\arctan \left(\frac{\left|G_{y}\right|}{\left|G_{x}\right|}\right)
$$

where $G_{x}$ and $G_{y}$ are the gradients in the $x$ and $y$ directions respectively.

If the edge strength between 8-connected neighborhoods, it will be removed. From the shown edges, the weak objects are improved by denoising with ALM and non-maximum suppression step. Thus, the number of strong objects is higher.

The hysteresis processing is to check the existence of weak edges in edge detection of medical images. Because the weak edges always are the component in strong edges combined with 8-connected neighborhoods when they are tracked. So, the weak edges will be presented in the detected results.

Finally, the results have many useful details. Here is the success of Canny; and it is continued in my algorithm. The time processing is shortened by ALM in the first step. The proposed method doesn't use a double threshold and filter to remove noise. That is to avoid the information of medical images when they are detected.

\section{Experiments and results}

As mentioned in section 3, this paper improves the quality of the Canny algorithm by ALM to remove noise at the smoothing step. The idea is to remove the usage of double threshold and filter of the previous Canny method. On the other hand, the proposed method also saves execution time. Because the time processing of other methods, using transform or combined threshold, is very slow.

The experiments were tested on different noise and blur levels of additive and multiplicative noise. My data set is a set of images that includes many types of medical images, such as CT, MRI, etc... This data set is free and available at http://www.barre.nom.fr/medical/samples/.

Two cases of strong and weak objects were tested; the size of testing images is $256 \times 256$. Each case calculated the number of edges that appear in the resulting image, and the timely processing of each method. The results of my proposed method are compared with other methods such as Canny (Canny, 1986), Cuckoo Search (Gonzalez, Castro, Melin, \& Castillo, 2015) and the scale multiplication wavelet transform (Zhang \& Bao, 2002). The proposed method has more edges than other methods, but the time processing is shorter. 


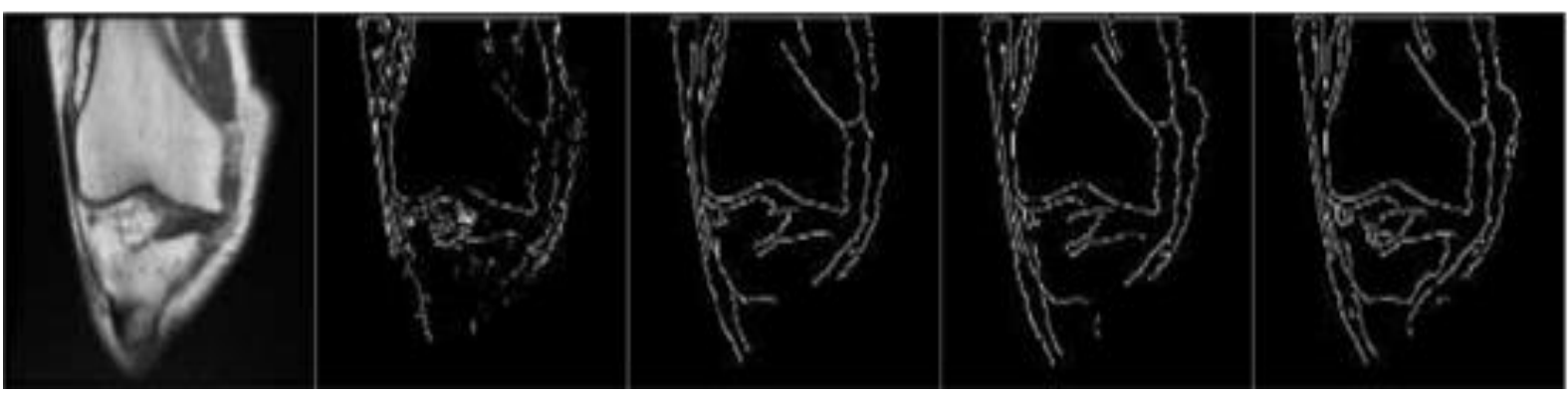

Figure 2. The results of edge detection by other methods with the strong object in medical image

Note: (a)The strong object in the original medical image.

(b)Edge detection by Canny method (3539 edges, execution time $\sim 1500$ seconds)

(c)Edge detection by Cuckoo search method (3603 edges, execution time $\sim 1850$ seconds)

(d)Edge detection by the scale multiplication wavelet transform (3745 edges, execution time $\sim 3700$ seconds)

(e)Edge detection by the proposed method (3879 edges, execution time $~ 1900$ seconds)

In this case, Figure 2(a) shows the original medical image. The results of Canny for edge detection are illustrated in Figure 2(b), the results of the Cuckoo search are illustrated in Figure 2(c), the results of scale multiplication wavelet transform are illustrated in Figure 2(d). Figure 2(e) shows the results of our proposed method. We can see the edge detection by the proposed method is better than the results shown in Figure 2(b), 2(c) and 2(d).

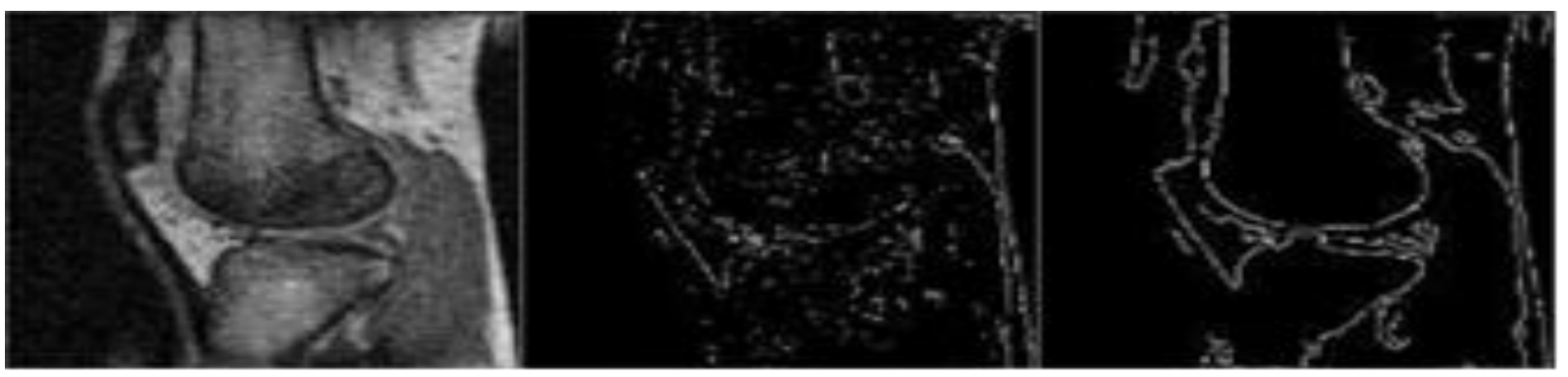

Figure 3. The results of edge detection by other methods with weak object in medical image

Note: (a) The weak object in the original medical image.

(b) Edge detection by Canny method (1905 edges, execution time 1700 seconds)

(c) Edge detection by Cuckoo search method (2973 edges, execution time $\sim 2200$ seconds)

(d) Edge detection by the scale multiplication wavelet transform (3236 edges, execution time 4500 seconds)

(e) Edge detection by the proposed method (4359 edges, execution time $~ 2100$ seconds)

In Figure 3, the medical image which has a weak object was tested. In Figure 3(a), the original medical image has a weak object. It is an object with boundaries that are blurred and noised. Nevertheless, the blur is a problem easily overcome by technical skill or the quality of the machine. The noise details are more popular than blur details. In this case, Gaussian noise and blur are added to medical images with the variance noise that is 0.00005 and the values of the point spread the function of Gaussian blur. The reason why Gaussian is selected with the plus noise and blur into pixels of medical images. They are very popular in medical images. 
The results of Canny for edge detection are illustrated in Figure 3(b), the results of the Cuckoo search are illustrated in Figure 3(c), the results of scale multiplication wavelet transform are illustrated in Figure 3(d). Figure 3(e) shows the results of our proposed method. We can see the edge detection by the proposed method is better than the results shown in Figure 3(b), 3(c) and 3(d).

From the results from Figure 2, Figure 3 and many other test cases, I conclude that the results of the proposed method are better than Canny, Cuckoo search and the scale multiplication wavelet transform in two cases: strong object and weak object. The number of edge detection which can be seen by naked eyes is higher than other methods. This paper proposes another evaluation which is to count the number of edges. This evaluation returns to the number of nonzero elements in the image matrix. The execution time of the proposed method is longer than Canny and Cuckoo search methods, but shorter than transform shown in Figure (d).

In edge detection for medical images, to avoid the loss of information, the algorithm should not use a filter or threshold to remove bad pixels. My proposed method improves the quality of the boundaries by the threshold in the first step of Canny, make them smoother and give more information than transform or multi-threshold. If the relationship between neighborhood and pixel, the edge strength, is not the largest, the algorithm can be removed. However, I can consider it if the strength is larger than over eight connected neighborhoods.

\section{Conclusion}

The adaptation of threshold and filter has many difficulties for image processing. This is a big problem for denoising or deblurring algorithms. Any denoising and deblurring methods successful, it is a good premise for edge detection, contour, or segmentation. The higher the number of edge detection is, the better the result of contour or segmentation is. In this paper, I propose a method to strengthen the quality of weak objects in order to improve the results of edge detection. My idea is to reconstruct the medical images which have noise and blur. The idea is based on the Augmented Lagrangian Method to improve the results of the Canny method. The results of the proposed method are compared with other methods such as Canny (Canny, 1986), Cuckoo Search (Gonzalez et al., 2015) and the scale multiplication wavelet transform (Zhang \& Bao, 2002). The results show that the proposed method detects more edges than other methods.

\section{References}

Bhatt, A. D., \& Warkhedkar, R. V. (2008). Reverse engineering of human body: A B-Spline based heterogeneous modeling approach. Computer-Aided Design and Applications, 5(1/4), 194-208.

Bhatt, A. D., \& Warkhedkar, R. V. (2009). Material-solid modeling of human body: A heterogeneous B-Spline based approach. Computer-Aided Design, 41(8), 586-597. 
Brigger, P., \& Unser, M. (1998). Multi-scale B-spline snakes for general contour detection. Wavelet Applications in Signal and Image Processing VI, SPIE, 3458, 92-102.

Canny, J. (1986). A computational approach to edge detection. Pattern Analysis and Machine Intelligence, IEEE Transactions on, PAMI, 8(6), 679-698.

Deriche, R. (1987). Using Canny's criteria to derive a recursively implemented optimal edge detector. International Journal of Computer Vision, 1, 167-187.

Easley, G., Labate, D., \& Lim, W. Q. (2008). Sparse directional image representations using the discrete shearlet transform. Applied and Computational Harmonic Analysis, 25(1), 25-46.

Gonzalez, C. I., Castro, J. R., Melin, P., \& Castillo, O. (2015). Cuckoo search algorithm for the optimization of type-2 fuzzy image edge detection systems. In IEEE Congress on Evolutionary Computation (CEC) (pp. 449-455). New York, NY: IEEE.

Marr, D., \& Hildreth, E. (1980). Theory of edge detection. Proceedings of the Royal Society, 207, 187-217.

Srishti. (2014). Technique based on Cuckoo's search algorithm for exudates detection in diabetic retinopathy. Ophthalmology research: An international journal, SCIENCEDOMAIN international, 2(1), 43-54.

Stanley, H., Khoshabeh, R., Kristofor, B. G., Philip, E. G., \& Truong, Q. N. (2011). An augmented lagrangian method for total variation video restoration. IEEE Transactions on Image Processing, 20(11), 3097-3111.

Strang, G. (1989). Wavelets and dilation equations: A brief introduction. SIAM Review, 31(4), 614-627.

Vincent, O. R., \& Folorunso, O. (2009). A descriptive algorithm for sobel image edge detection. Proceedings of Informing Science \& IT Education Conference (InSITE) (pp. 98-107).

Yuping, W., \& Yuanlong, C. (1995). Multiscale B-spline wavelet for edge detection. Science in China (Series A), 38(4), 499-512.

Zhang, L., \& Bao, P. (2002). Edge detection by scale multiplication in wavelet domain. Pattern Recognition Letters, 23(14), 1771-1784. 